\title{
Kink instabilities in jets from rotating magnetic fields
}

\author{
R. Moll, H. C. Spruit, and M. Obergaulinger
}

\author{
Max-Planck-Institut für Astrophysik, Karl-Schwarzschild-Str. 1, 85741 Garching, Germany \\ e-mail: rmo@mpa-garching.mpg.de
}

Received 4 July 2008 / Accepted 15 September 2008

\begin{abstract}
We have performed 2.5D and 3D simulations of conical jets driven by the rotation of an ordered, large-scale magnetic field in a stratified atmosphere. The simulations cover about three orders of magnitude in distance to capture the centrifugal acceleration as well as the evolution past the Alfvén surface. We find that the jets develop kink instabilities, the characteristics of which depend on the velocity profile imposed at the base of the flow. The instabilities are especially pronounced with a rigid rotation profile, which induces a shearless magnetic field. The jet's expansion appears to be limiting the growth of Alfvén mode instabilities.
\end{abstract}

Key words. magnetohydrodynamics (MHD) - instabilities - ISM: jets and outflows - galaxies: quasars: general - gamma rays: bursts

\section{Introduction}

Strong magnetic fields on large scales may play an essential, active role in the formation and evolution of jet-like outflows. The general idea is that a poloidal magnetic field, embedded in a plasma and anchored e.g. in an accretion disk or a black hole, is forced into rotation at the anchor point, a toroidal field develops and the plasma is accelerated by what can be interpreted as a centrifugal force in a corotating frame (Blandford \& Payne 1982; Spruit 1996; Königl \& Pudritz 2000). However, this magnetocentrifugal acceleration is only effective up until the Alfvén surface, defined as the surface where the flow velocity equals the Alfvén velocity. Beyond this point, the magnetic field will be strongly wound-up. Such a field configuration is potentially unstable with respect to certain MHD instabilities.

MHD jets are susceptible to a variety of instabilities. KelvinHelmholtz $(\mathrm{KH})$ instabilities are fed by the relative kinetic energy between the jet and the ambient medium. They can distort the jet surface only (ordinary modes) or the whole beam (e.g. Birkinshaw 1991), provoking shocks, mixing with ambient material and possibly a disruption of the jet (Bodo et al. 1995, 1998). The presence of strong magnetic fields, poloidal or toroidal, is expected to hamper the growth of $\mathrm{KH}$ instabilities (Appl \& Camenzind 1992; Keppens et al. 1999).

The free energy associated with the toroidal magnetic field is responsible for another class of instabilities, which is traditionally known as current-driven (CD) and of notorious importance in controlled fusion devices (for an introduction, see, e.g. Freidberg 1987; Bateman 1978). The relevance for magnetized astrophysical jets has been pointed out by Eichler (1993); Spruit et al. (1997); Begelman (1998) and others. Among CD instabilities, $m=1$ kink instabilities are the most effective. An ideal kink mode is characterized by helical displacements of the cylindrical cross sections of a plasma column. It is expected to grow on a dynamical time scale with respect to an Alfvén wave crossing the unstable column. The susceptibility is strongly dependent on the magnetic pitch, a measure for the degree of wind-up. Kink instabilities might destroy the ordered, symmetric state of a jet, leading to its disruption or, through magnetic reconnection, the associated dissipation of magnetic fields and steepening of the magnetic pressure gradient, to its acceleration (Drenkhahn 2002; Giannios \& Spruit 2006).

Different kinds of instability can mix and interact. For example, Baty \& Keppens (2002) show how CD instabilities can stabilize KH vortices at the jet surface. For this work, we used conditions under which $\mathrm{CD}$ kink instabilities are expected to dominate (low plasma- $\beta$, small magnetic pitch).

For a self-consistent study of kink instabilities, numerical simulations need to be carried out in 3D. Bell \& Lucek (1996) did so using a simple model in which a toroidal magnetic field configuration was allowed to expand into a uniform atmosphere. This generated a jet which was subject to kink instabilities. Nakamura \& Meier (2004) performed 3D simulations of MHD jets in variously stratified atmospheres, finding that they can develop kink-like distortions in the trans-Alfvénic region. Laboratory experiments of MHD jets have been performed by Hsu \& Bellan (2005), confirming that the magnetic pitch plays a crucial role for the formation of kink instabilities.

\subsection{Effects of jet expansion}

Jets from protostars, and especially AGN and microquasars, expand in width $d$ by orders of magnitude after passing through their Alfvén radius. In an expanding flow there is no clean separation between time dependence due to instability and that due to the expansion itself, making the question of stability less well defined. Analytical studies thus tend to focus on instabilities in a cylindrical geometry, with constant diameter (such as in the "magnetic tower" picture of Lynden-Bell 2003). Expansion has strong consequences on the behavior of instabilities, however, compared with jets modeled as cylinders of constant width.

First, there is the tendency for the toroidal (azimuthal, around the jet axis) component of the magnetic field to dominate in an expanding jet. From the induction equation, the poloidal and toroidal components of the field vary as $B_{\mathrm{p}} \sim 1 / d^{2}$ and $B_{\varphi} \sim 1 / d$ respectively (for constant jet velocity). Expansion thus causes a continual increase of the ratio $B_{\varphi} / B_{\mathrm{p}}$. Even when 
dissipation were to decrease the toroidal field at some point, the ratio increases again on further expansion. Free energy available in the toroidal field thus remains the dominant form of magnetic energy, and one may expect the question of stability and dissipation to remain relevant on all length scales. It also follows that the nonlinear development of instabilities in an expending jet is expected to be very different from the constant-diameter case.

Secondly, expansion has a strong effect even on the conditions for occurrence of instability. It has a stabilizing effect, since magnetic instabilities become ineffective when their signal speed (the Alfvén speed) drops below the lateral expansion speed of the jet. This is discussed further below.

\subsection{Rationale of the calculations}

The aim of the calculations reported here is to study how kink instability operates under these conditions of expansion of the jet over several orders of magnitude in width.

The degree of instability to be expected in a jet driven by a rotating magnetic field is intimately tied to the way it is collimated. If, instead of being cylindrical, the jet has a nonvanishing opening angle $\vartheta$, the expected incidence of instability depends on the details of the dependence of $\vartheta$ on distance $r$. An opening angle increasing with distance reduces instability, while for an asymptotically vanishing opening angle instability must always set in at some distance, if it was not present already from the start (see discussion in Sect. 1.3).

The setup in the simulations presented here produces jets in the intermediate case of an (approximately) constant opening angle: a "conical" outflow. It turns out that in this case the presence of instabilities and their amplitude depends on secondary conditions such as the rotation profile imposed at the base of the flow, hence it is a good test case for the incidence of instabilities.

Since the observed jets travel over such large distances, even marginal forms of instability can become effective. An important goal of the present calculations is therefore to cover a large range in distance, about 3 orders of magnitude. This is achieved by the use of a grid adapted to the approximately conical shape of the jet.

\subsection{Expected instability growth in expanding jets}

In the following, we estimate how the sideways expansion affects the growth of instabilities in broadening jets. In spherical coordinates $(r, \vartheta, \varphi)$, we assume that the jet radius (distance to the jet's central axis) is given by

$R=R^{\prime}\left(\frac{r}{r^{\prime}}\right)^{\alpha} \quad$ with $\quad R^{\prime}=r^{\prime} \sin \vartheta^{\prime}$

where the prime stands for evaluation at a reference distance $r^{\prime}$, for which we take a distance somewhat beyond the Alfvén radius. The flow has then approximately reached its asymptotic speed $v_{r} \approx$ const., and the magnetic field has become predominantly azimuthal. In the absence of magnetic dissipation due to instabilities, the field strength then varies as $B_{\varphi} \propto R^{-1}$ (magnetic flux conservation) and the density as $\rho \propto R^{-2}$ (mass conservation). Since the growth rate $\Gamma_{\mathrm{g}}$ is expected to scale with the Alfvén crossing rate $v_{\mathrm{A} \varphi} / R$, we introduce a dimensionless instability rate $\varkappa$ of order unity:

$\Gamma_{\mathrm{g}}=\varkappa \frac{v_{\mathrm{A} \varphi}}{R}=\varkappa \frac{v_{\mathrm{A} \varphi}}{R^{\prime}}\left(\frac{r}{r^{\prime}}\right)^{-\alpha}$ where $v_{\mathrm{A} \varphi}=B_{\varphi} / \sqrt{4 \pi \rho}$ is the azimuthal Alfvén velocity. The expansion rate is estimated by

$\Gamma_{\mathrm{e}}=\frac{\mathrm{d} \ln R}{\mathrm{~d} t}=\frac{1}{R} \frac{\mathrm{d} r}{\mathrm{~d} t} \frac{\mathrm{d} R}{\mathrm{~d} r}=\frac{\alpha v_{r}}{r}$

We find

$\frac{\Gamma_{\mathrm{g}}}{\Gamma_{\mathrm{e}}}=\frac{\varkappa}{v \alpha \sin \vartheta^{\prime}}\left(\frac{r}{r^{\prime}}\right)^{1-\alpha}$

with $v:=v_{r} / v_{\mathrm{A} \varphi} \approx$ const. according to the ballistic approximations mentioned above. Consequently, the instability growth rate dominates at some distance $r$ for a collimating jet $(\alpha<1)$. Decollimation $(\alpha>1)$, on the other hand, thwarts the growth of instabilities. A conical jet $(\alpha=1)$ constitutes a limiting case where all depends on the combination of parameters $x /\left(v \sin \vartheta^{\prime}\right)$, which is of order unity. A numerical simulation is necessary to find out whether the instability or expansion prevails.

The paper is organized as follows. In Sect. 2, we introduce the magnetocentrifugal jet model and account for the assumptions made in our simulations. A detailed description of the numerical setup, the coordinate system and the scale-free units employed in the analysis is given in Sect. 3. In Sect. 4 we give the parameters of the simulated cases and in Sect. 5 we present the results. There, we start by making predictions on the characteristics of instabilities by examining the relevant properties of our simulated jets. We proceed with an analysis of the instabilities that actually appeared and complete with looking for effects on the jets' dynamics. We finish with a discussion and conclusions in Sect. 6.

\section{The model}

The model is construed to apply to jets produced by ordered magnetic fields anchored in an accretion disk. This has become the default interpretation for the jets observed in AGN, microquasars and protostellar objects, though it must be kept in mind that observational evidence of the key ingredient in this model, the ordered field (Bisnovatyi-Kogan \& Ruzmaikin 1976; Blandford \& Payne 1982), is still somewhat indirect.

More uncertain is the shape of this field. The strength of the field anchored in the disk is likely to scale in some way with the orbital kinetic energy (or gas pressure) in the disk, hence will decline with distance $R$ from the rotation axis. In the absence of more detailed information, we consider a simple form for a field of this kind, one in which the vertical (normal to the disk) component at the surface $B_{z}$ varies as $B_{z}(R) \propto\left[1+\left(R / z_{0}\right)^{2}\right]^{-v}$. Neglecting gas pressure and fluid motions, the field above the disk would be a potential field, its shape defined uniquely by $B_{z}$. For $v=3 / 2$ it is the field of a monopole with the source at a depth $z_{0}$ below the center of the disk.

The initial state of the model is a gas distribution in hydrostatic equilibrium in a field of this monopolar shape. Rotation is applied at the lower boundary, in a region $R<R_{0}$ (see Sect. 3.3, for details). This generates an outflow with an approximately constant opening angle on the order $R_{0} / z_{0}$ (a "conical" outflow). The surrounding volume remains approximately in static equilibrium, and serves to collimate the outflow to the desired opening angle.

The magnetic field responds to the rotation by winding up. That is, a toroidal magnetic field $B_{\varphi}$ is produced and gives, together with the poloidal field $B_{r}$, rise to helical field lines. The magnetic pressure gradient (minus the tension force) associated 
with $B_{\varphi}$ gives rise to a poloidal acceleration of the plasma which is of centrifugal nature in a corotating frame. Beyond the Alfvén radius, the acceleration ceases to be effective, while the field becomes predominantly azimuthal. The further development depends on how strongly the jet is affected by instabilities in this highly wound-up field. Possibly, they endanger the jet's integrity and/or facilitate magnetic reconnection events. Magnetic field dissipation can entail further acceleration of the jet (Drenkhahn 2002). As discussed above (Sect. 1.3), the "conical outflow" produced in our monopolar background field is of special interest as it marks the boundary between cases expected to be strongly respectively weakly unstable.

A self-consistent investigation of the problem requires a full 3D treatment, because kink instabilities are non-axisymmetric. In addition to every 3D simulation we also performed an axisymmetric (2.5D) simulation with the same boundary and initial conditions. This way, we could detect whether the jet evolves differently due to the instabilities.

The basic parameters of the model are the magnitude of the rotation velocity, its profile $\Omega(R)$, the relative strength of the magnetic field as measured by plasma- $\beta$ of the initial state, and the jet's opening angle. The parameter values are chosen such that the Alfvén radius of the resulting outflow is located within the computational volume, so that the centrifugal acceleration process is covered in the simulation, but close to the lower boundary, so that the subsequent evolution can be followed over as large a distance as numerically feasible. Increasing the imposed rotation rate moves the Alfvén radius toward the lower boundary. Due to numerical limitations, however, $v_{\varphi}$ could not be increased indefinitely in the simulations, and a compromise was necessary. In the results reported below the region inside the Alfvén radius occupies about 10-20\% of the box length.

\section{Methods}

We employ a spherical grid for our jet simulations. This enables us to follow jets with opening angles over a much longer distance than is possible with a Cartesian grid, because the jet need not be overresolved at large heights in order to properly resolve its base. The obvious choice of letting the jet propagate along the polar axis is numerically problematic if non-axisymmetric flows are involved, because the grid is singular there. We therefore let the jet flow in equatorial direction. The computational volume covers a range $\Delta \theta=\Delta \phi$ in the polar and azimuthal angles, adjusted to the opening angle of the flow.

\subsection{MHD equations and numerical solver}

We numerically solved the ideal adiabatic MHD equations, including a temperature-dependent temperature-control term $K=$ $K(T(t))$. Explicitly, the equations are:

$$
\begin{aligned}
& \frac{\partial \rho}{\partial t}+\boldsymbol{\nabla} \cdot(\rho \boldsymbol{v})=0, \\
& \frac{\partial \boldsymbol{v}}{\partial t}+\boldsymbol{v} \cdot \boldsymbol{\nabla} \boldsymbol{v}=-\frac{1}{\rho} \boldsymbol{\nabla} p+\frac{1}{4 \pi \rho}(\boldsymbol{\nabla} \times \boldsymbol{B}) \times \boldsymbol{B}-\boldsymbol{\nabla} \Phi, \\
& \frac{\partial e}{\partial t}+\boldsymbol{\nabla} \cdot\left[\left(e+p+\frac{B^{2}}{8 \pi}\right) \boldsymbol{v}-\frac{1}{4 \pi} \boldsymbol{B}(\boldsymbol{B} \cdot \boldsymbol{v})\right]=-\rho \boldsymbol{v} \cdot \boldsymbol{\nabla} \Phi+K, \\
& \frac{\partial \boldsymbol{B}}{\partial t}=\boldsymbol{\nabla} \times(\boldsymbol{v} \times \boldsymbol{B}),
\end{aligned}
$$

where

$$
e=\frac{1}{2} \rho v^{2}+\frac{B^{2}}{8 \pi}+\frac{p}{\gamma-1}
$$

is the total energy density, $\gamma=5 / 3$ is the adiabatic index, $p$ is the gas pressure, $\Phi$ is the gravitational potential (external, no self-gravity) and the other symbols have their usual meanings. A notorious problem with low- $\beta$ MHD simulations in fully conservative form, as in the code used here, is the amplification of discretization errors that occurs because the gas pressure is only a small contribution to the total energy (cf. (9)), which is dominated by the magnetic energy. As in the case of highly supersonic flows, these errors manifest themselves in the form of "negative pressures" at occasional grid points. This problem does not occur when an equation for the thermal energy equation is used instead of the total energy. We compute, in parallel, an alternative update of the gas pressure from the thermal energy equation, in the form

$$
\frac{\partial p}{\partial t}+\boldsymbol{\nabla} \cdot(p \boldsymbol{v})=-(\gamma-1) p \boldsymbol{\nabla} \cdot \boldsymbol{v}+K
$$

Where negative pressures appear, they are replaced by this value.

Another device that turns out very useful to avoid negative pressures is the temperature-control term $K$ in Eq. (7). For this we use a scheme loosely modeled after Newtonian cooling, or an optically thin radiative loss process. After every full time step, we add/subtract thermal energy according to

$\frac{\Delta p(t)}{p(t=0)}=-\frac{T(t)-T(t=0)}{T(t=0)} \frac{\Delta t}{\tau_{K}}$

where $T$ is the temperature and $\tau_{K}$ is a time scale chosen so as to keep the temperature within about a factor 30 of the initial atmospheric value.

With the MHD Poynting vector

$\boldsymbol{S}=-\frac{1}{4 \pi}(\boldsymbol{v} \times \boldsymbol{B}) \times \boldsymbol{B}$,

Eq. (7) can also be written as

$\frac{\partial(e+\rho \Phi)}{\partial t}+\boldsymbol{\nabla} \cdot\left[\left(\frac{1}{2} \rho v^{2}+\frac{\gamma}{\gamma-1} p+\rho \Phi\right) \boldsymbol{v}+\boldsymbol{S}\right]=K$,

describing the change of total energy including gravitational potential energy. We will employ this form later when we look at the energy flow rates.

We used a newly developed Eulerian MHD code (Obergaulinger et al., in preparation) to solve Eqs. (5)-(8). It is based on a flux-conservative finite-volume formulation of the MHD equations and the constraint transport scheme to maintain a divergence-free magnetic field (Evans \& Hawley 1988). Using high-resolution shock capturing methods (e.g., LeVeque 1992), it employs various optional high-order reconstruction algorithms and approximate Riemann solvers based on the multi-stage method (Toro \& Titarev 2006). The simulations presented here were performed with a fifth order monotonicitypreserving reconstruction scheme (Suresh \& Huynh 1997), together with the HLL Riemann solver (Harten 1983) and third order Runge-Kutta time stepping.

\subsection{Grid coordinates}

In the 3D simulations, our computational domain was centered around the $y$-axis in a "standard" spherical coordinate system $(r, \theta, \phi)$ with $\theta$ being the polar angle from the $z$-axis and $\phi$ being the azimuthal angle about the $z$-axis in the $x$-y-plane. See Fig. 1 for an illustration. Positioning the domain in equatorial (rather than polar) direction yields a grid which is free of singularities 


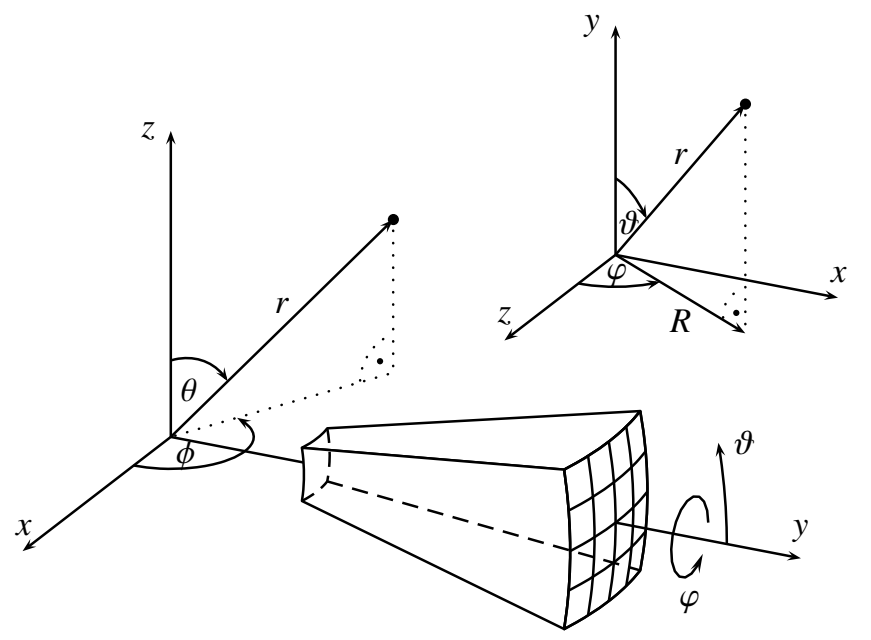

Fig. 1. Computational domain and coordinate nomenclature (schematic drawing). The jet propagates along the $y$-axis $(\theta=\phi=\pi / 2)$, near which the grid is quasi-Cartesian in the normal plane. To describe the results of the simulations, we use the alternate spherical coordinate system $(r, \vartheta, \varphi)$ shown in the upper right inset, which takes the $y$-axis as the polar axis.

and quasi-regular in transverse jet direction: Near the $y$-axis, the distance between two $\phi=$ const. curves is $\Delta x \approx r \Delta \phi$ if we neglect terms of order $(\theta-\pi / 2)^{2}$ and of order $(\phi-\pi / 2)^{3}$. The distance between two $\theta=$ const. curves is then $\Delta z \approx r \Delta \theta$. With uniform spacings $\Delta \theta$ and $\Delta \phi$, we thus obtain a grid whose area elements are approximately those of an equidistant Cartesian grid in a plane normal to the $y$-axis.

For data analysis and plotting we use the "alternative" spherical coordinate system $(r, \vartheta, \varphi)$ with $\vartheta$ being the polar angle from the $y$-axis and $\varphi$ being the azimuthal angle about the $y$-axis in the $z$ - $x$-plane. It is adapted to the propagation direction of the jet and as such better suited to describe its physics. $R=r \sin \vartheta$ denotes the distance to the $y$-axis. Generally, we refer to the $r$-direction with "poloidal" or "radial", the $\varphi$-direction with "toroidal" or "azimuthal" and the $y$-axis as "polar axis" or "central axis".

In the $2.5 \mathrm{D}$ simulations, the jet propagates along the $z$-axis and the $\phi$-direction is taken to be symmetric. However, to avoid confusion, we use the same nomenclature as in the 3D simulations throughout this paper. That is, we visualize the jet as propagating in $y$-direction and employ the $(r, \vartheta, \varphi)$ system for describing its physics.

For a proper resolution at all radii, it turned out to be necessary to employ logarithmic grid spacing (Park \& Petrosian 1996) in $r$-direction. The $r$-left interface of grid cell $i$ is situated at

$r_{1}^{i}=r_{1}^{0}\left(\frac{r_{r}^{n-1}}{r_{1}^{0}}\right)^{i / n}$

where $n$ is the total number of cells in the domain which is bounded by $r=r_{1}^{0}$ and $r=r_{r}^{n-1}=r_{1}^{n}$. The cell center of grid cell $i$ is situated at $r_{\mathrm{c}}=\left(r_{1}^{i}+r_{r}^{i}\right) / 2$.

\subsection{Initial and boundary conditions}

The initial magnetic field is

$\boldsymbol{B}(r)=\frac{g}{r^{2}} \hat{\boldsymbol{e}}_{r}$, corresponding to a magnetic monopole of charge $g$ located at the coordinate origin. The associated vector potential

$\boldsymbol{A}=\frac{g}{r} \tan \left(\frac{\theta}{2}\right) \hat{\boldsymbol{e}}_{\phi}$

was employed in the numerical setup to ensure solenoidality of the discretized magnetic field. Satisfying $\boldsymbol{\nabla} \times \boldsymbol{B}=0$, the initial magnetic field is force-free.

We impose the static gravitational field of a point mass $M$, also located at the origin. The stratification of gas pressure in this potential is chosen such that the plasma $-\beta:=p / p_{\text {mag }}$ in the initital state is constant throughout the computational domain. Hence, since $B \propto r^{-2}, p \propto r^{-4}$. The density in the initial state is determined from hydrostatic equilibrium: $\rho G M=-r^{2} \mathrm{~d} p / \mathrm{d} r \propto r^{-3}$ where $G$ is the gravitational constant. The temperature, sound speed and Alfvén velocity vary as $T \propto r^{-1}, c_{\mathrm{S}} \propto r^{-1 / 2}$ and $v_{\mathrm{A}} \propto r^{-1 / 2}$ in this stratification.

The lower boundary of the computational volume, where the jet's "nozzle" resides, is at a distance $r_{\mathrm{n}}$ from the origin. The conditions at this surface are related to the gravitational potential by

$\Phi(r)=-\frac{G M}{r} \quad$ with $\quad M=\frac{4 p_{\mathrm{n}} r_{\mathrm{n}}}{G \rho_{\mathrm{n}}}$,

where the subscript $\mathrm{n}$ (for "nozzle") denotes the values at $r_{\mathrm{n}}$.

At the sides $(\theta$ and $\phi)$ and top (upper $r$ ) of the domain, we use open boundaries which allow for an almost force-free outflow of material: $p, \rho$, all components of $\boldsymbol{v}$ and the transverse components of $\boldsymbol{B}$ are mirrored across the boundary interface to the opposing "ghost cells", the normal component of $\boldsymbol{B}$ is determined by the solenoidality condition. The open boundaries work well and cause only minimal artefacts in the form of reflections. At the bottom (lower $r$ ) of the domain, where the jet emanates, $p$ and $\rho$ are kept fixed at their initial values in all ghost cells. The magnetic field is determined by extrapolation from the interior of the domain using the same scheme as for open boundaries. The velocity is prescribed to be zero except for the ghost cells below the nozzle area $\left(R \leq R_{\text {jet,n }}\right.$ at $\left.r=r_{\mathrm{n}}\right)$. There, an azimuthal velocity field $\boldsymbol{v}=v_{\varphi} \hat{\boldsymbol{e}}_{\varphi}$ is maintained, with either a Keplerian velocity profile

$v_{\varphi}= \begin{cases}v_{\varphi, \mathrm{n}}^{\max } \sqrt{\frac{0.2 R_{\mathrm{jet}, \mathrm{n}}}{R}} & \text { for } 0.2 R_{\mathrm{jet}, \mathrm{n}} \leq R \leq R_{\mathrm{jet}, \mathrm{n}} \\ 0 & \text { elsewhere }\end{cases}$

or a rigid rotation profile

$v_{\varphi}= \begin{cases}v_{\varphi, \mathrm{n}}^{\max } \frac{R}{R_{\mathrm{jet}, \mathrm{n}}} & \text { for } \quad R \leq R_{\mathrm{jet}, \mathrm{n}} \\ 0 & \text { elsewhere. }\end{cases}$

Note that we use the term "Keplerian" to indicate only that $v_{\varphi} \propto R^{-1 / 2}$. The central mass $M$ only serves to balance our chosen stratification and is not to be understood as the center of an accretion disk.

\subsection{Units}

The setup described above is unambiguously determined by 6 parameters, $B_{\mathrm{n}}, p_{\mathrm{n}}, \rho_{\mathrm{n}}, R_{\text {jet,n }}, r_{\mathrm{n}}$ and $v_{\varphi, \mathrm{n}}^{\max }$, but they are not all independent. As units of length, pressure and density we use $l_{0} \equiv 2 R_{\text {jet, } \mathrm{n}}, p_{0} \equiv p_{\mathrm{n}}$ and $\rho_{0} \equiv \rho_{\mathrm{n}}$. The physical quantities expressed in these units are listed in Table 1 . Since these units are arbitrary, the number of independent parameters defining the problem reduces to $6-3=3$. These are a plasma- $\beta$ value (which 
Table 1. Normalization units.

\begin{tabular}{ccc}
\hline \hline Quantity & Symbol(s) & Unit \\
\hline length & $x, y, z, r, R, h$ & $l_{0}$ \\
gas pressure & $p$ & $p_{0}$ \\
density & $\rho$ & $\rho_{0}$ \\
velocity & $v$ & $c_{\mathrm{s} 0}=\sqrt{\gamma p_{0} / \rho_{0}}$ \\
time & $t, \tau$ & $t_{0}=l_{0} / c_{\mathrm{s} 0}$ \\
energy density & $e$ & $p_{0}$ \\
energy flow rate & $\mathcal{E}$ & $p_{0} l_{0}^{3} / t_{0}$ \\
force density & $F$ & $p_{0} / l_{0}$ \\
magnetic flux density & $B$ & $B_{0}=\sqrt{8 \pi p_{0}}$ \\
current density & $j$ & $j_{0}=B_{0} c /\left(4 \pi l_{0}\right)$ \\
\hline
\end{tabular}

determines $\left.B_{\mathrm{n}}\right)$, an opening angle $\vartheta_{\mathrm{o}}:=\arcsin \left(l_{0} / 2 r_{\mathrm{n}}\right)$, and a Mach number for the rotation: either $v_{\varphi, \mathrm{m}}^{\max } / c_{\mathrm{s}, \mathrm{n}}$ or $v_{\varphi, \mathrm{n}}^{\max } / v_{\mathrm{A}, \mathrm{n}}$.

The sound speed, Alfvén velocity and escape velocity at $r=$ $r_{\mathrm{n}}$, expressed in the normalization units, are $c_{\mathrm{s}, \mathrm{n}}=c_{\mathrm{s} 0}$,

$v_{\mathrm{A}, \mathrm{n}}=\sqrt{\frac{2}{\gamma}} \frac{B_{\mathrm{n}}}{B_{0}} c_{\mathrm{s} 0} \approx 1.1 \frac{B_{\mathrm{n}}}{B_{0}} c_{\mathrm{s} 0}$

and $v_{\mathrm{esc}, \mathrm{n}}=\sqrt{\frac{8}{\gamma}} c_{\mathrm{s} 0} \approx 2.2 c_{\mathrm{s} 0}$.

For the sake of clarity, we usually omit the normalization unit. For example, $v=5$ would denote a velocity of $5 c_{\mathrm{s} 0}$, which is sonic Mach 5 at the jet nozzle.

\section{Cases studied}

In the following we present the results of two 3D simulations for two different rotation profiles imposed at the nozzle, the Keplerian and rigid rotation profiles given by (18, case K3) and (19, case R3). These are compared with two 2.5D simulations with the same initial and boundary conditions (cases K2 and R2).

In all cases, the initial state has a constant plasma- $\beta$ of $1 / 9$ $\left(B_{\mathrm{n}}=3\right)$, the maximum rotation velocity at the boundary is $v_{\varphi, \mathrm{n}}^{\max }=0.33 c_{\mathrm{S}, \mathrm{n}}=0.1 v_{\mathrm{A} r, \mathrm{n}}$ and the initial (half) opening angle is $\vartheta_{\mathrm{o}}=5.7^{\circ}\left(r_{\mathrm{n}}=5\right)$. This choice of parameters yields a jet with a magnetic pitch low enough to be unstable to kinks. At the same time, it avoids numerical problems found to arise with higher (supersonic) rotation velocities as a boundary condition. The "grid noise" in the 3D simulations (the grid is not axisymmetric in the rotation direction $\varphi$ ) turned out to be sufficient to excite instabilities, so we did not need to apply a perturbation by hand. For the temperature-control term, we used $\tau_{K}=2$.

In the 3D simulations, we used 384 logarithmically spaced grid cells in radial direction and 96 uniformly spaced grid cells in each of the two angular directions. The physical extent of the simulated domain was 500 in the radial direction and $33.8^{\circ}$ in each angular direction. The ratio between the maximum and minimum $r$ is $505 / 5=101$. The $2.5 \mathrm{D}$ simulations were performed with the same resolution in the radial direction and 64 grid cells in the evolved angular direction which had an extent of $16.9^{\circ}$. The 3D simulations each ran for about one week (wall clock time) on 64 processors with MPI parallelization.

\section{Results}

The jets are initially accelerated mainly by gas pressure. This holds up to the sonic surface, which lies about halfway to the

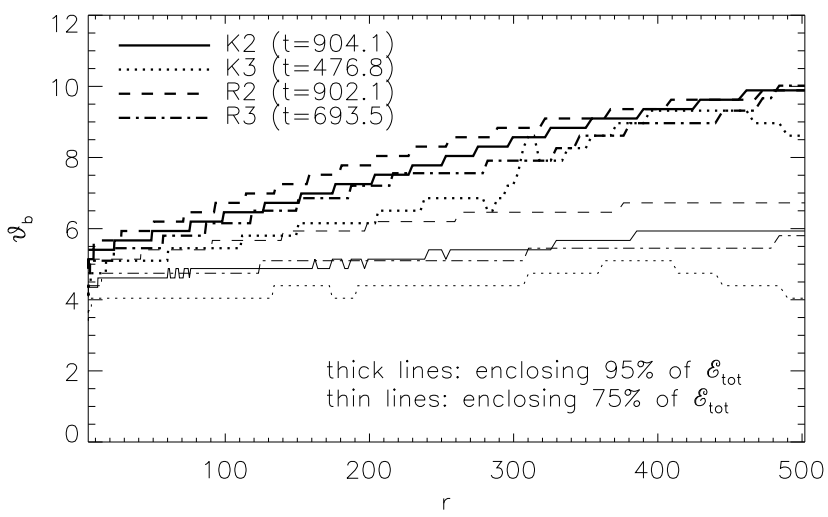

Fig. 2. Jet border, defined as the polar angle within which a given percentage of energy flows (see Eq. (26) for the definition of $\mathcal{E}_{\text {tot }}$ ), as a function of distance. In all cases, most of the energy flow is contained within the $\vartheta \approx \vartheta_{\mathrm{o}}=5.7^{\circ}$ surface, where $\vartheta_{\mathrm{o}}$ is the initial opening angle. A minor but increasing amount of energy flows outside this angle. The energy density in the jet (especially the toroidal field) causes it to decollimate somewhat compared with the conical configuration in which it is embedded.

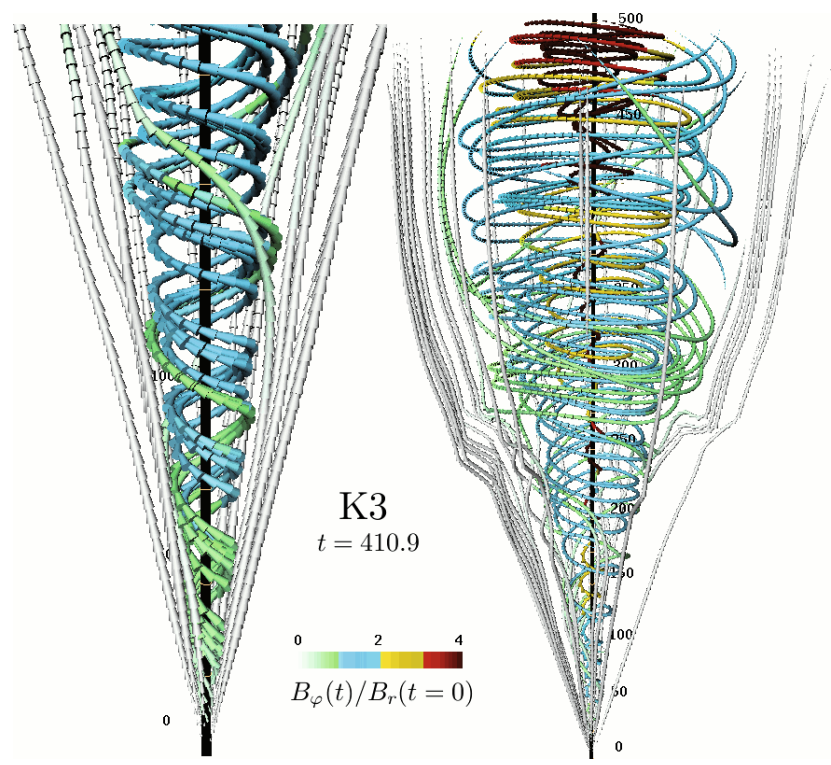

Fig. 3. Selected magnetic field lines in the $3 D$ simulation with a Keplerian velocity profile. The right-hand plot shows the entire domain $(r=5 \ldots 505)$, the left-hand plot only the lower part up to $r \approx 200$. The color coding represents the strength of the azimuthal magnetic field. The magnetic field lines, which were purely radial to begin with, wind many times around the central axis, rendering it susceptible to kink instabilities.

Alfven surface. Then, the Lorentz force becomes the dominant driving force. The Alfvén radii are at $r \approx 30 \ldots 140$, depending on the simulation and the direction $\vartheta$ : near the axis (small $\vartheta$ ), the poloidal field $B_{r}$ is amplified and the Alfvén radii are at larger distances than in the outer regions (large $\vartheta$ ), where $B_{r}$ is attenuated. The opening angle of the jets increase somewhat with distance, but to a first approximation the flow can still be treated as conical, see Fig. 2 . The jet front crosses the upper boundary $(r=505)$ at $t \approx 260$ in the $2.5 \mathrm{D}$ simulations and at $t \approx 330$ in the $3 \mathrm{D}$ simulations. The latter are subject to more numerical dissipation of kinetic energy, because the grid there is not symmetric in azimuthal direction. This reduces the injected Poynting flux, see Fig. 11. Apart from that, 2.5D and 3D simulations give, for our purposes, comparable results. Figure 3 shows how the 


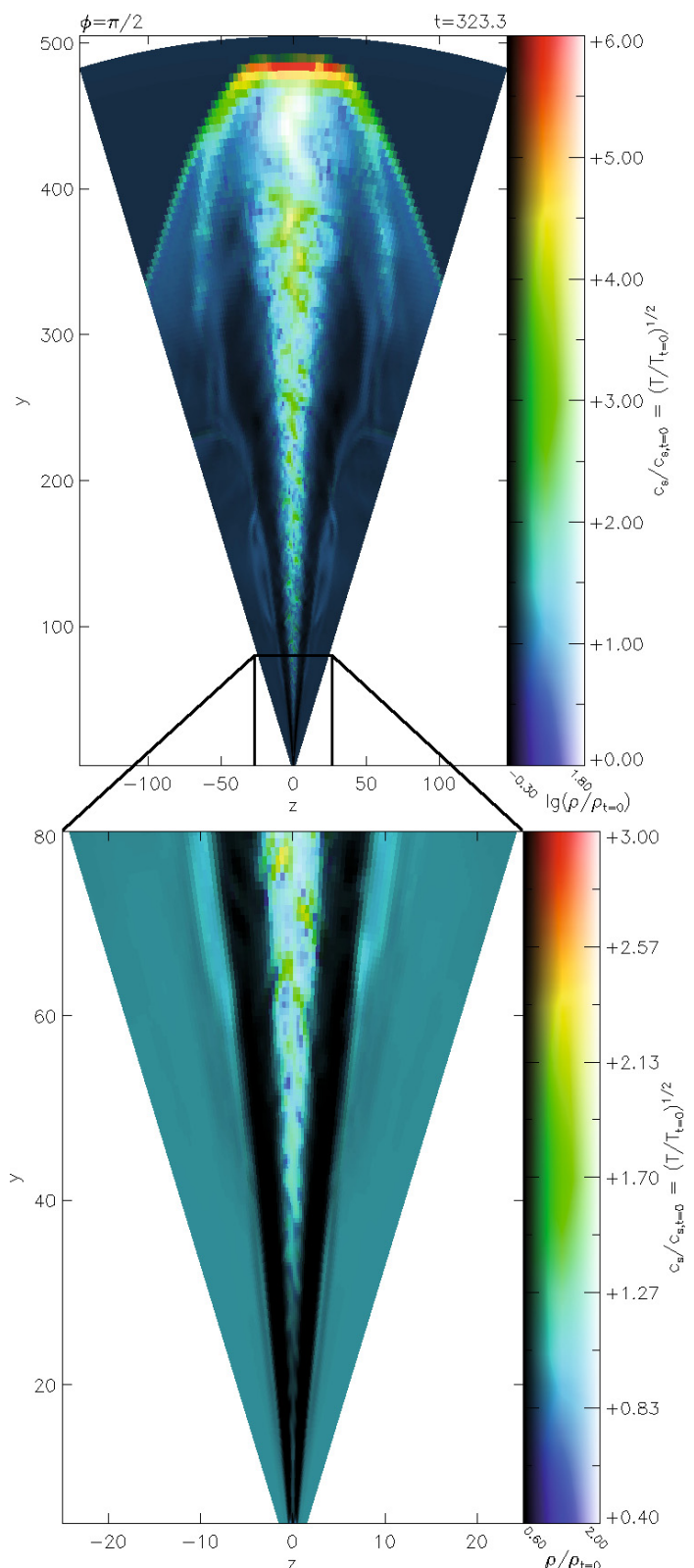

Fig. 4. Density and temperature in a meridional slice in simulation K3 (Keplerian rotation profile, 3D). The density is encoded in intensity, with bright colors representing regions that are overdense with respect to the environment. The (square root of the) temperature is encoded in the hue, with blue meaning cold and red meaning hot with respect to the environment. The hoop stress associated with $B_{\varphi}$ squeezes the plasma towards the central axis and creates an underdense cavity around the central part of the jet. At the boundary of this cavity the environment exerts the stress that confines the jet. The observed opening angle for a jet like this would be smaller than the width of the cavity.

magnetic field is wound up inside the jet in one of the simulations. A typical density and temperature distribution is shown in Fig. 4.

\subsection{Expected instabilities}

The observed instabilities can be compared with expectations from linear stability theory. To do this, we extract from the axisymmetric simulations the quantities that enter the stability conditions, and then compare the result with the evidence of
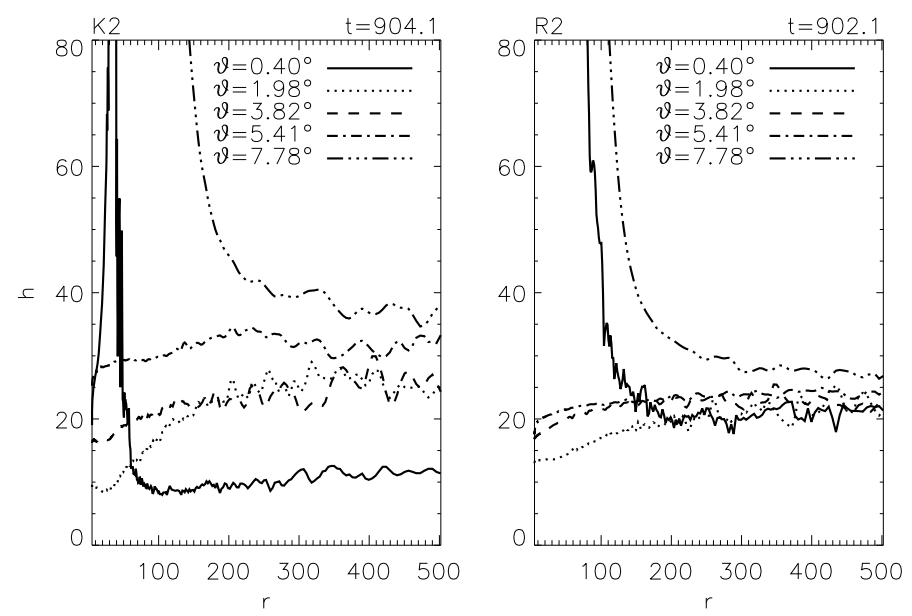

Fig. 5. Magnetic pitch as a function of distance in the 2.5D simulations. The magnetic pitch is also the smallest possible wavelength of an instability. The dependence of $h$ on the polar angle $\vartheta$ is stronger in the Keplerian case (left plot), suggesting higher stability.

non-axisymmetric instability in the corresponding 3D simulation. The available stability conditions apply only to steady or static configurations and have been derived either in the context of controlled fusion or cylindrical jets (Appl et al. 2000; Lery et al. 2000), hence the comparison can only be indicative.

According to the Kruskal-Shafranov criterion, the longitudinal wavelength of an instability must be at least as high as the magnetic pitch on the unstable surface, defined to be the distance covered during one revolution of a helical field line about the central axis. Besides being the result of linear stability analyses in the context of controlled fusion, it can be derived heuristically from geometric arguments (Johnson et al. 1958). Therefore, it should give a convenient scale also in cases for which it was not originally intended, like the expanding jets studied here. Deviations can be expected e.g. from the effect of one-ended line-tying, which in some cases has been found to lead to increased instability as opposed to a configuration without a free end (Furno et al. 2006; Lapenta et al. 2006; Sun et al. 2008).

For a conical jet, the magnetic pitch is

$h=2 \pi R\left|\frac{B_{r}}{B_{\varphi}}\right|$

on the $\vartheta=$ const. surface. See Appendix A for a derivation of this expression. In the simulations, $h$ decreases with $r$ and settles to a constant value above the Alfvén radius, see Fig. 5 (compare also Fig. 3). The variation of the pitch with $\vartheta$ depends on the kind of rotation imposed at the lower boundary. In the Keplerian case, the dependence is strong, with the asymptotic pitch being approximately 10 near the axis and 40 at the limb of the jet. In the rigid rotation case, $h \approx 25$ in all directions within the jet.

The Alfvén crossing time in a conical, unaccelerated jet, defined as the time it takes an azimuthal Alfvén wave to orbit the central axis, is given by

$\tau_{\mathrm{c}}=\frac{\iota R}{v_{\mathrm{A} \varphi}-\iota v_{R}}$

where $\iota=2 \pi$ for a full revolution, $v_{\mathrm{A} \varphi}=$ const. is the azimuthal Alfvén speed and $v_{R}=v_{r} \sin \vartheta$ is the expansion velocity. In the simulations, $v_{\mathrm{A} \varphi} \approx$ const. above the Alfvén radius. This is as expected theoretically from conservation of mass and magnetic 

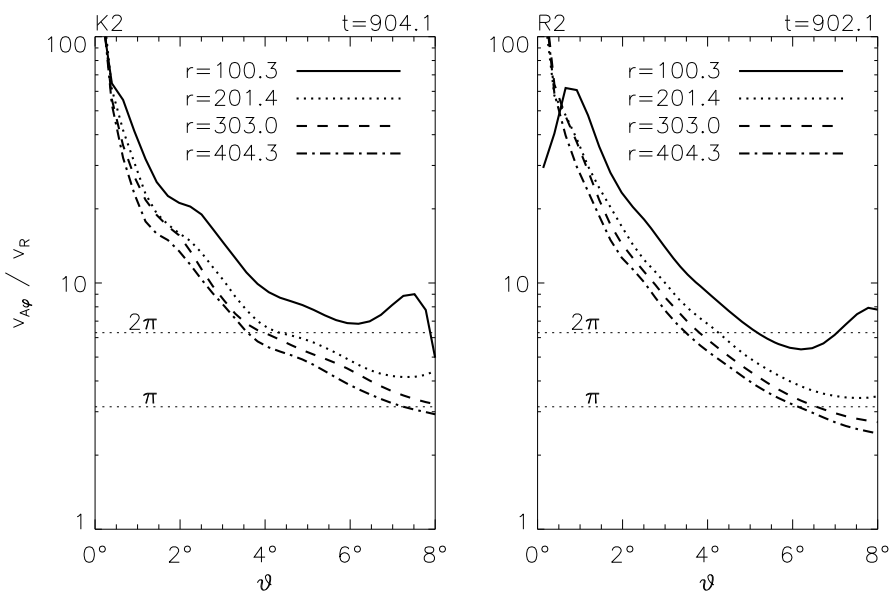

Fig. 6. The expected onset of instability depends on the ratio of the azimuthal Alfvén speed and the expansion velocity. This ratio is shown here for the 2.5D simulations. The horizontal dotted lines are for two estimates of the condition under which growth is possible (see text). Below the respective line, expansion prevails and an instability cannot grow.
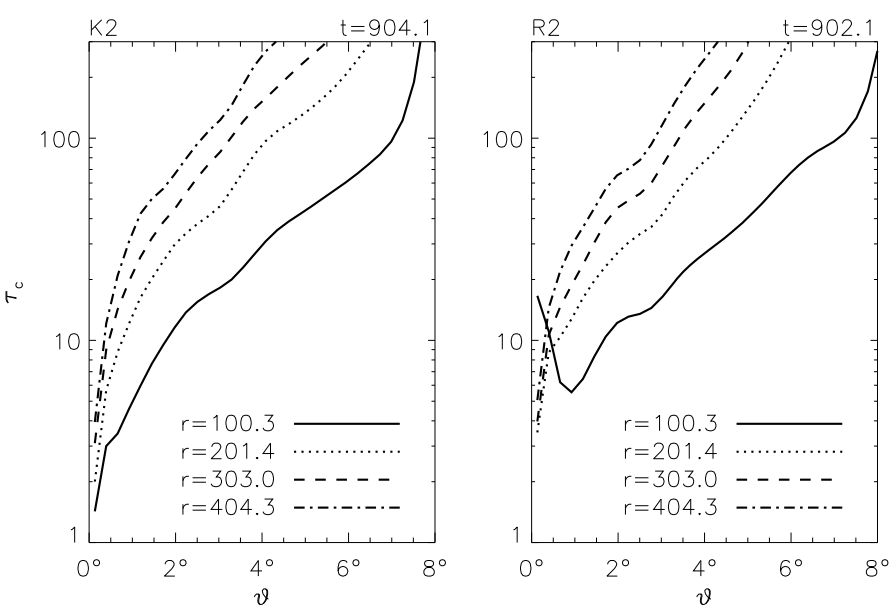

Fig. 7. Alfvén crossing times with $\iota=\pi$ in the 2.5D simulations. In both cases, $\tau_{\mathrm{c}}$ and with it the expected instability growth time increases with radius and distance from the central axis. The curves for $r \approx 100$ represent an underestimate, because the jet still accelerates at this distance.

flux in a conically expanding, steady axisymmetric jet. $\tau_{\mathrm{c}}$ is finite and physically meaningful only if the condition

$\frac{v_{\mathrm{A} \varphi}}{v_{R}}>\iota$

is satisfied. If it is not, the expansion takes place too fast for an Alfvén wave to cross the jet and an Alfvén mode instability cannot grow. While the critical value of $\iota$ is arguable, we note that causal contact across the jet by Alfvén waves is only possible if $\iota \geq \pi$. The situation in our simulations is illustrated in Figs. 6 and 7. Instabilities can grow only slowly on magnetic surfaces with large $\vartheta$. Depending on the $\iota$ needed for efficient growth, they may even be stalled due to the jet's expansion. In any case, instabilities grow most rapidly if they start at small $r$. In regions where the jet is accelerating $\left(\mathrm{d} v_{r} / \mathrm{d} r>0\right)$ or decollimating $(\mathrm{d} \vartheta / \mathrm{d} r>0$ along a field line), the effective Alfvén crossing time is underestimated by Eq. (23). The jet is then stabler than condition (24) suggests.

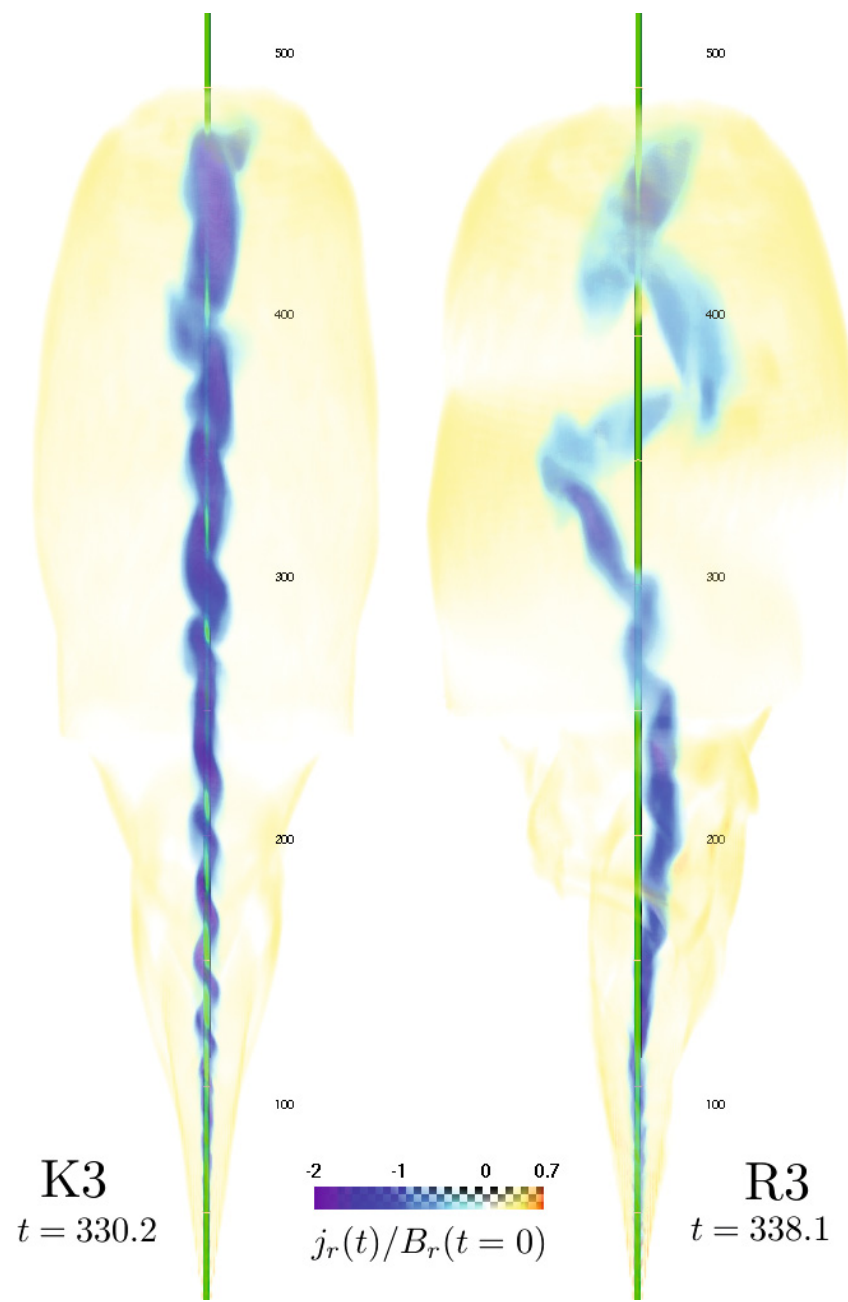

Fig. 8. Radial component of the current density $(\boldsymbol{\nabla} \times \boldsymbol{B})$ in the 3D simulations just before the jets reach the upper boundary. The rod in the middle of the jets is their central axis ( $y$-axis). Helical distortions, characteristical for kink instabilities, can be seen in the backward current (blue) in both cases. The amplitudes and wavelengths are significantly larger in the simulation with a rigid rotation profile (right-hand image).

\subsection{Instabilities found in the simulation}

We observe non-axisymmetric, kink-like distortions in the magnetic field and other quantities in both 3D simulations. They emerge near the Alfvén radius, propagate with the flow and grow in amplitude along the way. It is convenient to look at the current density $\boldsymbol{j}=\frac{c}{4 \pi} \boldsymbol{\nabla} \times \boldsymbol{B}$ for a quantitative analysis. The radial component $j_{r}$ is related to $B_{\varphi}$ and is as such characteristic for the distortions in the magnetic field. In the unperturbed case, it is concentrated about the central axis and along the outer boundary of the cavity illustrated in Fig. 4, with respectively opposite orientation. In our simulations, $B_{\varphi}$ is directed in negative $\varphi$-direction and the axial current, accordingly, in negative $r$-direction. We denote this backward current with $j_{r}^{-}$, so that $j_{r}=j_{r}^{+}+j_{r}^{-}$.

In the rigid rotation case (R3), the distortions attained large amplitudes of several degrees. Looking at $B_{\varphi}$ in the $r=$ const. plane, we find that the whole jet is affected by the kink. The number of visible radial nodes is $2-4$, corresponding to wavelengths on the order of 150 , i.e. several times larger than the magnetic pitch. Owing to the distortions in the magnetic field, the axial current was perturbed as shown in Fig. 8 on the right-hand image. $j_{r}^{-}$helically twines around the central axis in 

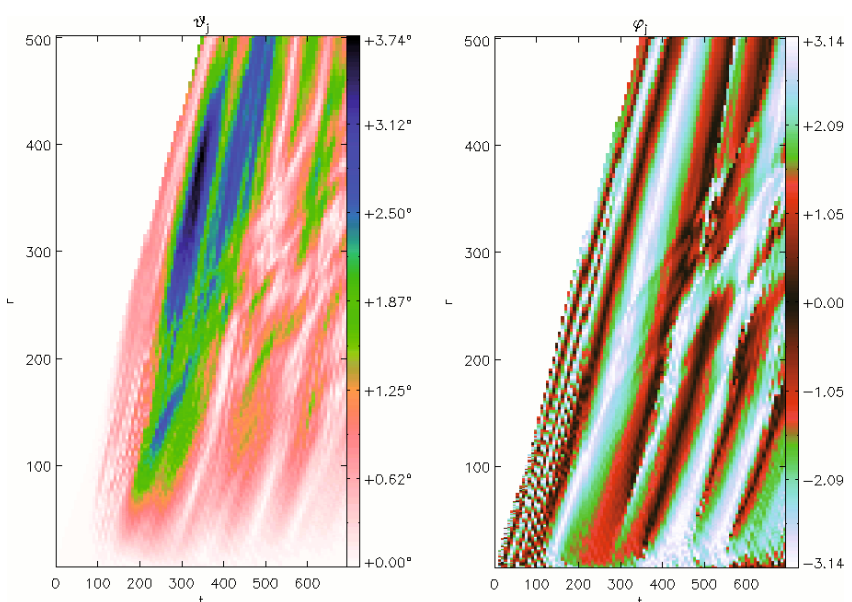

Fig. 9. Position of the barycenter of the backward current (blue material in Fig. 8) in simulation R3. The left-hand map shows the amplitude of the instabilities and the right-hand one its phase. The blank region is where the jet has not been yet, its border marks the jet front. Observers moving with the flow follow a time-position curve which is (approximately) parallel to the front, i.e. the instabilities are at rest with respect to a comoving frame.

reminiscence of "ideal" kink instabilities with an azimuthal mode number $m=1$.

To analyze the unstable displacements, we determine the barycenter of the backward current $j_{r}^{-}$in the $r=$ const. plane, denoting its location with $\left(\vartheta_{j}, \varphi_{j}\right)$. The result, from which one can directly read off amplitudes and wavelengths, is shown in Fig. 9. The slope of the points of constant phase $\varphi_{j}$ in the $r-t$ diagram corresponds with the flow velocity $v_{r}$. That is, the instabilities are at rest with respect to a comoving frame. We estimate the growth time in such a frame by introducing an observer moving with flow and measure $\vartheta_{j}$ in doing so. We find strictly increasing, exponential growth if the observer is located just behind the jet front, see upper panel in Fig. 10. The exponential growth time $\tau_{\mathrm{g}}$ is generally on the order of the Alfvén crossing times shown in Fig. 7. For observers which are farther behind the jet front, the amplitude does not follow a simple exponential increase, see lower panel in Fig. 10 for an example. Rather, it saturates and even declines in some cases. The reason for this is not clear. We cannot rule out the possibility that there is stabilizing feedback from the upper boundary. Considering that the flow is super-Alfvénic there, this seems unlikely though.

In the Keplerian rotation case (K3), the jet also exhibits kink-like distortions, see left-hand image in Fig. 8. However, the perturbation amplitudes are much smaller, with $\vartheta_{j}$ attaining peak values of about $1.4^{\circ}$ directly behind the jet front and only about $0.5^{\circ}$ farther behind. Unlike in the rigid rotation case, only inner regions of the jet are affected by the kinks, the jet border is relatively unharmed. The wavelengths are on the order of 25-50, i.e. there are more radial nodes than in the rigid rotation case. Even for an observer traveling just behind the jet front, the amplitude is not strictly increasing, but saturates and tapers off after an initial rise with a growth time of $\tau_{\mathrm{g}} \approx 50$, i.e. also on the order of the relevant Alfvén crossing times in Fig. 7.

\subsection{Impact on dynamics and energetics}

Instabilities release magnetic energy by transforming it into kinetic energy, but for dissipation in the sense of magnetic reconnection sufficiently small length scales have to develop. In
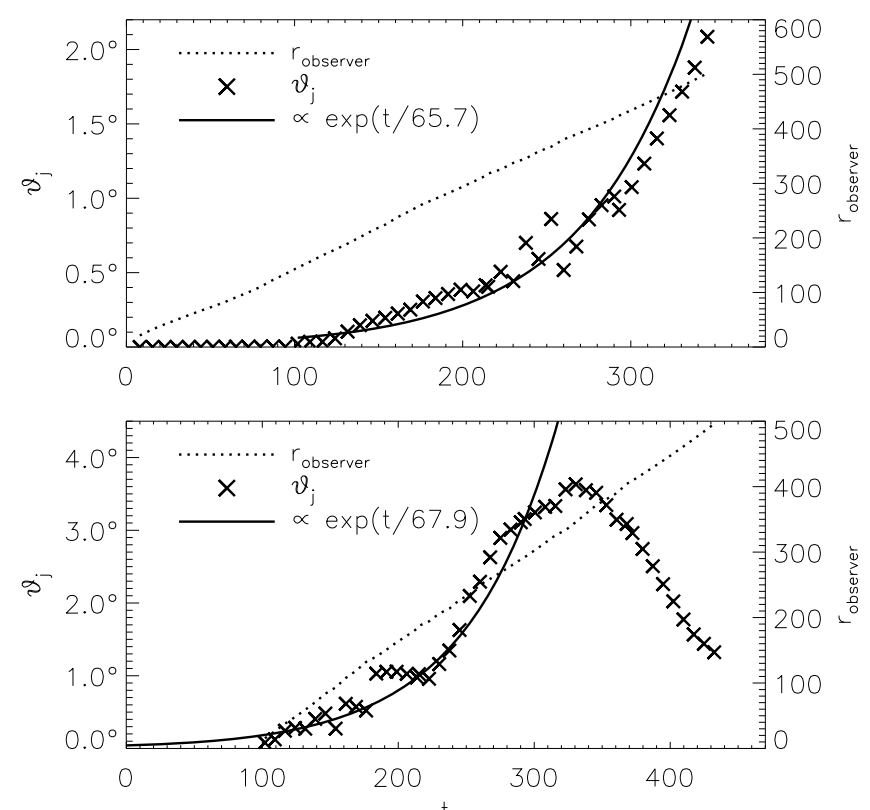

Fig. 10. Amplitude of the perturbations (crosses, left-hand axis) as seen by an observer (dotted line, right-hand axis) located just behind the jet front (upper panel) and located 130 length units behind the jet front (lower panel) in simulation R3. The exponential fits (solid line, lefthand axis) yield the instability growth time.

ideal MHD simulations like ours, such dissipation is present in the form of numerical diffusion due to the effect of interpolations across adjacent grid cells. The effect cannot be modeled by Ohmic resistivity but can be quantified through secondary effects like changes in the energy fluxes.

We did not find conclusive evidence of magnetic field dissipation provoked by the instabilities in the 3D simulations. For example, the flow of nonradial magnetic field

$$
\int_{r=\text { const. }} \sqrt{B_{\vartheta}^{2}+B_{\varphi}^{2}} v_{r} \mathrm{~d} A
$$

shows asymptotically the expected $\sim r$ behavior (as $B_{\varphi} \sim r^{-1}$ and $A \sim r^{2}$ ) with minor fluctuations but no decreasing trend when compared to the $2.5 \mathrm{D}$ simulations.

It is helpful to look at the energy fluxes. From Eq. (13), the energy flow rate in poloidal direction is

$\mathcal{E}_{\text {tot }}(t, r)=\int_{r=\text { const. }}\left[\left(\frac{1}{2} \rho v^{2}+\frac{\gamma}{\gamma-1} p+\rho \Phi\right) v_{r}+S_{r}\right] \mathrm{d} A$.

Decomposing the integral from left to right, we identify the flow rates of kinetic energy $\mathcal{E}_{\text {kin }}$, thermal enthalpy $\mathcal{E}_{\text {thrm }}$, gravitational potential energy $\mathcal{E}_{\text {grav }}$ and magnetic enthalpy $\mathcal{E}_{\text {mag }}$. We plotted these in Fig. 11 for the simulations R3 and R2. The total energy flow $\mathcal{E}_{\text {tot }}$ rises for small $r$ due to the temperature-control term $K$ in Eq. (7). The conversion of Poynting flux to kinetic energy flux in the 3D simulation looks qualitatively the same as in the 2.5D comparison simulation. In particular, there is no evidence of an additional conversion of $\mathcal{E}_{\text {mag }}$ to $\mathcal{E}_{\text {kin }}$ due to magnetic field dissipation. This agrees with the fact that there is no additional acceleration of the jets, see Fig. 12.

\section{Discussion and conclusions}

We have simulated magnetocentrifugally driven, conical jets over a range in distance of 1000 times the initial jet radius, in 


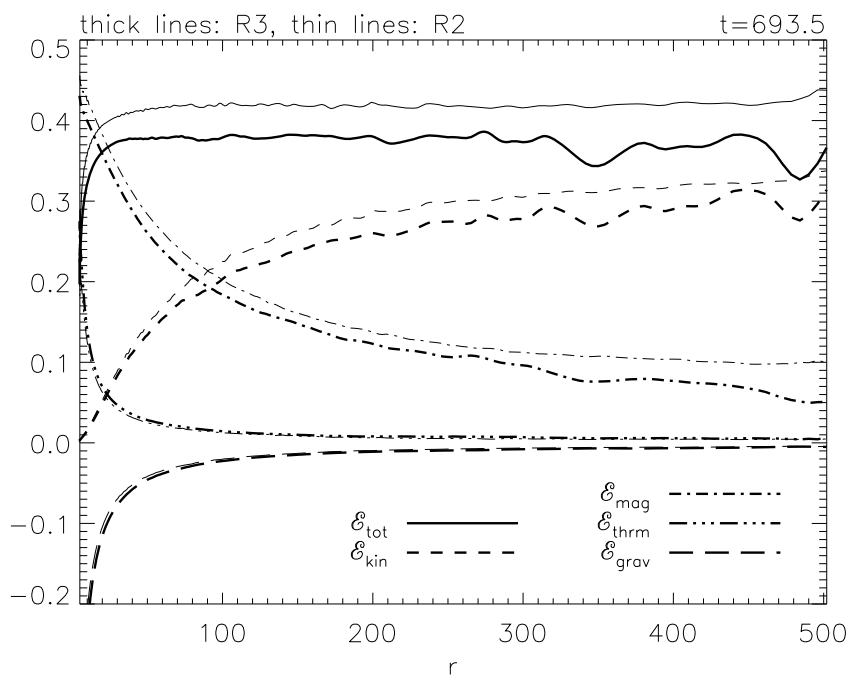

Fig. 11. Energy flow rates through $r=$ const. in 3D (thick lines) and in the $2.5 \mathrm{D}$ (thin lines) simulations with a rigid rotation profile. There is no clear evidence of additional conversion of Poynting flux to kinetic energy. The energy flow through the lateral boundaries is virtually zero at all times. The situation is similar in the Keplerian case (K3 and K2).
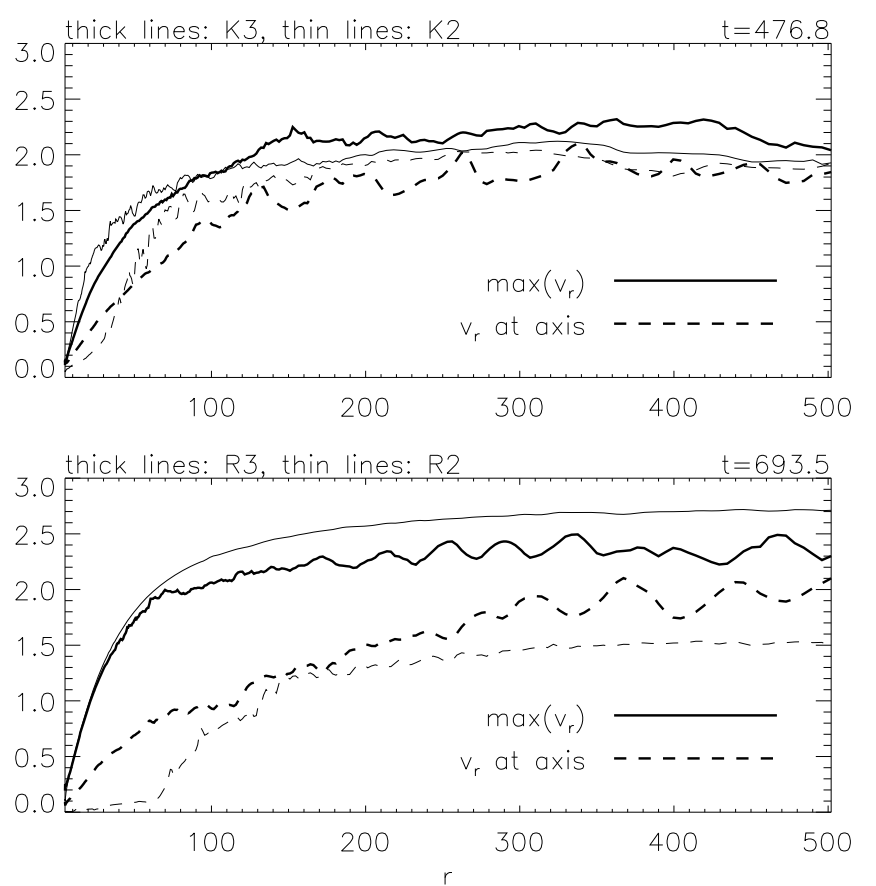

Fig. 12. Radial velocities in the $3 \mathrm{D}$ (thick lines) and in the 2.5D (thin lines) simulations. The jets do not accelerate above the Alfvén radius, despite instabilities. The value of $v_{r}$ near the central axis in simulation R3, though rising, does not give conclusive evidence of acceleration, because the jet's axis is strongly distorted by the instabilities.

both $3 \mathrm{D}$ and axisymmetric $2.5 \mathrm{D}$. The calculations extend to a factor of about 5-10 beyond the Alfvén surface. The 3D jets developed non-axisymmetric instabilities of the kink kind.

The violence of the instabilities depends on the rotation profile applied at the base. With a rigid rotation $(\propto R)$ profile, the perturbations grow to much larger amplitudes than with a Keplerian $\left(\propto R^{-1 / 2}\right)$ profile. We suspect that the reason for the differing behavior lies in the magnetic shear, defined as the variation of the magnetic pitch with distance to the axis. In the rigid rotation case, there is virtually no shear as opposed to the Keplerian case, for which the pitch increases with distance from the axis, see Fig. 5. A shear-free configuration is expected to be unstable to non-resonant modes, whereas a configuration with increasing pitch is expected to be unstable to modes with a resonant surface inside the jet (Appl et al. 2000; Lery et al. 2000). This fits well with what we observe in the simulations, viz. that the kink is confined inside the jet in the Keplerian case. Heuristically speaking, the differing behavior could be attributed to the fact that the outer (high $\vartheta$ ) layers of the jet, which are more stable (higher magnetic pitch), damp internally arising modes in the Keplerian case.

In both cases, the longitudinal wavelength of the instabilities is $\sim 5$ times larger than the value of the magnetic pitch near the axis. The relation is qualitatively consistent with the findings of Appl et al. (2000) for a cylindrical jet. The growth time of the instabilities is on the order of the Alfvén crossing time. The exact relation is difficult to determine, because the crossing time as well as the location of the resonant surface can only be estimated. As the azimuthal magnetic field strength and with it the azimuthal Alfvén speed decrease past the Alfvén surface, opposing parts of the jets become causally disconnected from each other. Thus, the jet expands too fast for Alfvén mode instabilities to grow. The effect is amplified if the jet is diverging. Recollimation, on the other hand, should boost the growth of instabilities.

As found in other studies, the conversion of magnetic enthalpy (Poynting flux) to kinetic energy is fairly efficient, on the order $70 \%$. Dissipation of magnetic fields by internal instabilities is expected to contribute additional acceleration of the flow (Drenkhahn 2002). The calculations do not show a clear signature of this process. It seems that either the observed region is too small, and/or the numerical dissipation of magnetic fields is too weak. Also, from a macroscopic point of view, the instabilities were not violent enough to bring together fields with an antiparallel component, as is necessary for magnetic reconnection to occur. Moreover, most of the magnetic enthalpy was already converted in the magnetocentrifugal acceleration process. Therefore, even if there was magnetic dissipation, the effect would not be dramatic. Nevertheless, we found that the magnetic field gets significantly distorted by the instabilities. This should facilitate magnetic field dissipation further downstream but it may be necessary to extend the calculations to larger distances to see the effect.

It is tempting to compare the instability-related structures in the simulations with structures in observed jets. The $3 \mathrm{D}$ jet structure in Fig. 4, for example, is reminiscent of the semi-regular patterns seen in $\mathrm{H} \alpha$ images taken of outflows from young stellar objects (YSO) like HH 34 (Reipurth et al. 2002). There are, however, other possible interpretations of the observed structure. The wiggles in YSO jets could also be the result of a precessing or orbitally moving source (Masciadri \& Raga 2002, and references therein). The symmetric nature of structures often seen in jet and counterjet (e.g. HH 212 Wiseman et al. 2001) suggests a modulation of the outflow speed or mass flux originating at the source of the outflow rather than an instability developing further away. The irregularities caused by the instabilities studied here are possibly more important for internal magnetic energy release inside the jet than for major observable structures like the knots and wiggles in YSO jets, though they are likely to contribute to these at some level as well.

Acknowledgements. We thank an anonymous referee for constructive comments. We are also grateful to Dimitrios Giannios for stimulating discussions. 


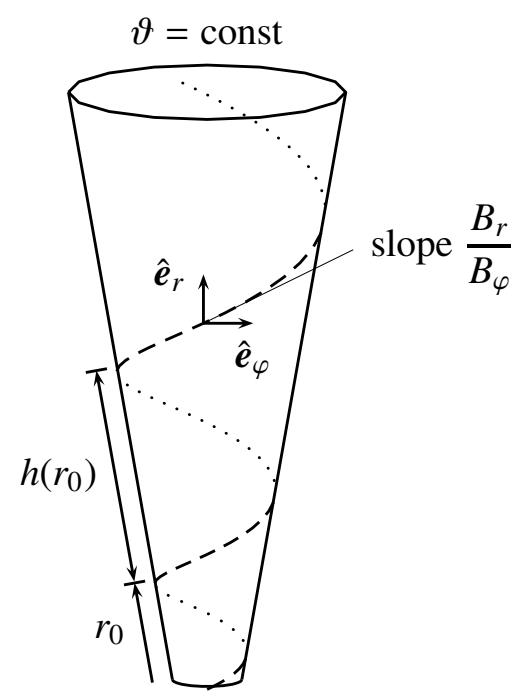

Fig. A.1. Magnetic field line with pitch $h$ on a conical surface.

\section{Appendix A: Magnetic pitch for a conical jet}

The radial progress of the field line depicted in Fig. A.1 is determined by

$$
\frac{\mathrm{d} r}{R \mathrm{~d} \varphi}=\left|\frac{B_{r}}{B_{\varphi}}\right|=: a(r)
$$

where $R=r \sin \vartheta$ is the distance to the polar axis and $a(r)$ is the unsigned slope. Assuming that $B_{r} \propto r^{-2}$ and $B_{\varphi} \propto r^{-1}$ due to magnetic flux conservation, we can write

$a(r)=a_{0} \frac{r_{0}}{r}$.

By integrating the resulting expression we obtain the radial distance covered after one revolution:

$$
\int_{r_{0}}^{r_{0}+h} \mathrm{~d} r=\int_{0}^{2 \pi} a_{0} R_{0} \mathrm{~d} \varphi \Rightarrow h=2 \pi a_{0} R_{0} .
$$

Alternatively, we could also define a local magnetic pitch $\tilde{h}$ by taking $a=a_{0}=$ const. for the slope. The result is

$$
\frac{\tilde{h}}{r_{0}}=\exp \left(2 \pi a_{0} \sin \vartheta\right)-1 \text {. }
$$

The difference between $h$ and $\tilde{h}$ turned out to be insignificant in our analysis. This is understandable since $\tilde{h} \rightarrow h$ for small $a_{0} \sin \vartheta$.

\section{References}

Appl, S., \& Camenzind, M. 1992, A\&A, 256, 354

Appl, S., Lery, T., \& Baty, H. 2000, A\&A, 355, 818

Bateman, G. 1978, MHD Instabilities (MIT Press)

Baty, H., \& Keppens, R. 2002, ApJ, 580, 800

Begelman, M. C. 1998, ApJ, 493, 291

Bell, A. R., \& Lucek, S. G. 1996, MNRAS, 283, 1083

Birkinshaw, M. 1991, in Beams and Jets in Astrophysics, ed. P. Hughes (Cambridge, UK: Cambridge University Press), 278

Bisnovatyi-Kogan, G. S., \& Ruzmaikin, A. A. 1976, Ap\&SS, 42, 401

Blandford, R. D., \& Payne, D. G. 1982, MNRAS, 199, 883

Bodo, G., Massaglia, S., Rossi, P., et al. 1995, A\&A, 303, 281

Bodo, G., Rossi, P., Massaglia, S., et al. 1998, A\&A, 333, 1117

Drenkhahn, G. 2002, A\&A, 387, 714

Eichler, D. 1993, ApJ, 419, 111

Evans, C. R., \& Hawley, J. F. 1988, ApJ, 332, 659

Freidberg, J. P. 1987, Ideal Magnetohydrodynamics (Plenum Press)

Furno, I., Intrator, T. P., Ryutov, D. D., et al. 2006, Phys. Rev. Lett., 97, 015002

Giannios, D., \& Spruit, H. C. 2006, A\&A, 450, 887

Harten, A. 1983, J. Comput. Phys., 49, 357

Hsu, S. C., \& Bellan, P. M. 2005, Physics of Plasmas, 12, 2103

Johnson, J. L., Oberman, C. R., Kulsrud, R. M., \& Frieman, E. A. 1958, Phys. Fluids, 1, 281

Keppens, R., Tóth, G., Westermann, R. H. J., \& Goedbloed, J. P. 1999, J. Plasma Phys., 61, 1

Königl, A., \& Pudritz, R. E. 2000, Protostars and Planets IV, 759

Lapenta, G., Furno, I., Intrator, T., \& Delzanno, G. L. 2006, J. Geophys. Res., Space Phys., 111, 12

Lery, T., Baty, H., \& Appl, S. 2000, A\&A, 355, 1201

LeVeque, R. J. 1992, Numerical Methods for Conservation Laws, 2nd Ed., ETH Zürich: Lectures in mathematics (Birkhäuser)

Lynden-Bell, D. 2003, MNRAS, 341, 1360

Masciadri, E., \& Raga, A. C. 2002, ApJ, 568, 733

Nakamura, M., \& Meier, D. L. 2004, ApJ, 617, 123

Park, B. T., \& Petrosian, V. 1996, ApJS, 103, 255

Reipurth, B., Heathcote, S., Morse, J., Hartigan, P., \& Bally, J. 2002, AJ, 123, 362

Spruit, H. C. 1996, ArXiv Astrophysics e-prints

Spruit, H. C., Foglizzo, T., \& Stehle, R. 1997, MNRAS, 288, 333

Sun, X., Intrator, T. P., Dorf, L., Furno, I., \& Lapenta, G. 2008, Phys. Rev. Lett., 100,205004

Suresh, A., \& Huynh, H. 1997, J. Comput. Phys., 136, 83

Toro, E. F., \& Titarev, V. A. 2006, J. Comput. Phys., 216, 403

Wiseman, J., Wootten, A., Zinnecker, H., \& McCaughrean, M. 2001, ApJ, 550, L87 